\title{
Fluctuation in salivary alpha-amylase activity and vital signs during dental implant surgery
}

\author{
Afnan Sabbagh ${ }^{1}$, Hidemi Nakata ${ }^{1 *}$, Ahmed Abdou², Shohei Kasugai ${ }^{1}$ and Shinji Kuroda ${ }^{1 *}$
}

\begin{abstract}
Background: Salivary alpha-amylase (SAA) activity is thought to be an indicator of mental stress. However, the relationship between SAA activity and mental stress in patients undergoing dental implant treatment has not been investigated. The present study aimed to examine the correlation between SAA activity and changes in the patient's vital signs during dental implant surgery.

Results: SAA activity was higher after surgery compared to the pre-surgical measurements. A significant positive correlation was observed between sAA activity and heart rate $(\mathrm{HR})\left(r_{s}=0.434, p=0.007\right)$, as well as the peripheral blood oxygen $\left(\mathrm{SpO}_{2}\right)\left(r_{s}=0.392, p=0.016\right)$.

Conclusion: SAA activity and the patient's stress levels tended to increase after the surgical procedure. A positive correlation was observed between $\mathrm{SpO}_{2}$ and sAA activity. A significant positive correlation was also observed between the between the HR and SAA activity, although there was no correlation between blood pressure and sAA activity. Thus, sAA may be a valuable indicator of the stress and anxiety accumulated during dental implant surgery.
\end{abstract}

Keywords: Salivary alpha-amylase, Stress, Patients, Anxiety, Implant surgery, Stress hormone, Vital signs

\section{Background}

Oral rehabilitation with dental implants is a widely used clinically effective and predictable modality for patients with complete or partial edentulism [1-3]. In addition to the cornerstone implant placement surgery, dental implant treatment requires different surgical techniques, such as bone augmentation, soft tissue management, or a second surgery to deliver the healing abutment, depending on each individual case [4]. These procedures may induce psychological and physiological stress in patients, which can be controlled in several cases, so that patients exhibit normal clinical conditions before and during surgical implant treatment $[5,6]$. However, mental astress, such as

\footnotetext{
* Correspondence: hidemi.irm@tmd.ac.jp; skuroda.mfc@tmd.ac.jp 'Department of Oral Implantology and Regenerative Dental Medicine, Division of Oral Health Sciences, Graduate School of Medical and Dental Sciences, Tokyo Medical and Dental University, 1-5-45 Yushima, Bunkyo-ku, Tokyo 113-8510, Japan

Full list of author information is available at the end of the article
}

sadness, anger, uneasiness or fear, and nervousness, can cause an elevation in blood pressure (BP) (i.e., hypertension), which can result in the cancellation of implant surgeries $[7,8]$. Moreover, the surgical procedure may be cancelled owing to the incidence of a hypertensive episode immediately before the commencement of the procedure, despite the pre-surgical measurements being normal and a negative history of medical conditions. Unforeseen sudden cancellations are inconvenient to both patients and clinicians; upon enquiry, patients mention that they were anxious before surgery [9].

$\mathrm{BP}$ is controlled by the autonomic nervous system, which includes the sympathetic nervous system (SNS) and parasympathetic nervous system (PSNANS); in other words, the stress level in an individual is modulated by the balance between the PSNANS and SNS [10, 11].

Increasing stress levels induce SNS predominance, preparing the body for the "fight or flight" response, 
thereby increasing BP by increasing the heart rate (HR) and inducing contraction of the peripheral blood vessels.

Since anxiety is a form of psychological stress, an increase in anxiety results in a surge in stress levels [12, 13]. The human body reacts automatically in response to stress. Therefore, evaluating anxiety and/or stress levels in patients would be useful to ensure patient safety during treatment. Various questionnaires have been used to assess the degree of anxiety that have been shown to have simple application in routine clinical practice.

Cortisol levels in the peripheral blood have been used to evaluate stress levels, since the increase in stress promotes the secretion of cortisol from the adrenal glands $[14,15]$. Furthermore, studies have reported that alphaamylase activity in the saliva also increases with the increase in stress $[16,17]$. The measurement of salivary alpha-amylase (sAA) activity is currently possible within less than $1 \mathrm{~min}$, whereas the measurement of cortisol requires more time. Thus, the measurement of sAA would be advantageous for evaluating stress levels in patients in routine dental practice.

One study showed that sAA activity increased significantly when patients were exposed to a stressful condition compared to a relaxed condition [18]. Another study reported a significant correlation between the State/Trait Anxiety Inventory score and alpha-amylase levels [19].

The present study aimed to examine the anxiety and stress levels of patients who underwent dental implant surgery based on the activity of sAA before and after the procedure, in addition to the evalution of their vital signs. The purpose of this study was to examine the correlation between sAA activity, as an efficient stress biomarker for anxiety, and stress levels, as measured by the changes in the vital signs, such as BP, HR, and oxygen level, in patients undergoing dental implant surgery. The measurement of sAA activity acted as an adjunctive indicator for stress and anxiety and we assessed the relationship and correlation between sAA and these vital signs to test this hypothesis.

\section{Materials and methods Design and setting}

This clinical study incorporated a prospective, non-controlled, non-interventionist design and was conducted at Tokyo Medical and Dental University's Dental Hospital. Patients were recruited from the outpatient clinic.

\section{Ethical considerations}

This study was approved by the ethics committee of Tokyo Dental and Medical University (approval numbers: D2020-027 and D2020-028).

\section{Participants}

A total of 44 patients who underwent surgery for dental implant placement were recruited, including 28 women and 16 men. The participants' mean age was 62 years. We divided patients into the following (two) main groups, depending on the type of implant surgery: the first surgery $(n=19)$ and second implant $\operatorname{surgery}(n=22)$ groups. Only three patients underwent connective tissue graft placement. However, the final sample size was reduced to 40 patients, as three patients were excluded because of incorrect data and the fourth patient cancelled the procedure due to a hypertensive crisis.

The exclusion criteria for this study were as follows: (1) uncontrolled diabetes, (2) pregnancy or nursing, (3) substance abuse, (4) very heavy smoking habit ( $\geq 11$ cigarettes/day), (4) treatment with intravenous aminobisphosphonates, (5) severe bruxism, (6) untreated periodontitis, (7) poor oral hygiene, and (8) untreated mental disorders. The purpose of the study was explained to all patients, and consent was obtained.

Salivary amylase activity and vital sign measurement Salivary amylase activity was measured using a handheld salivary amylase monitor (NIPRO, Osaka, Japan). This analyzer enables automatic and time-efficient measurement of salivary amylase activity using a dry-chemical system, within approximately $1 \mathrm{~min}$ from the collection of saliva to the completion of measurement. The tip of the testing strip was placed under the tongue for $30 \mathrm{~s}$ to collect a sufficient amount of saliva so that it would completely cover the testing strip. Thereafter, it was removed from the mouth, and the other end of the tip was pulled slowly until it clicked and the testing strip was immediately inserted into the device. The plastic arm was pulled upwards, setting off a 10-s countdown on the device's screen. Subsequently, the testing strip was pulled partially until it clicked again, and the result was displayed on the screen after an additional $10 \mathrm{~s}$.

The tip of the testing strip was soaked in a buffer containing 2-chloro-4-nitrophenyl-4-O-beta-D-galactopyranosylmaltoside (Gal-G2-CNP), which acts as a substrate and chromogen, and dried. One-unit activity (U) per mass of enzyme is defined as the amount of enzyme whose activity produces $1 \mu \mathrm{mol}$ of the reducing sugar maltose in $1 \mathrm{~min}$ [20].

We also measured all vital signs of all patients with the hospital monitor [HR, BP (Table 1), peripheral blood oxygen saturation $\left(\mathrm{SpO}_{2}\right)$, and body temperature] at three time-points: (1) before, (2) during, and (3) after surgery.

The first sAA sample was obtained and analyzed immediately before surgery, i.e., prior to the administration of local or intravenous anesthesia. Another sample was obtained immediately after the completion of surgery and before the patient rinsed his/her mouth. 
Table 1 Reference values of blood pressure $(\mathrm{mmHg})$

\begin{tabular}{ll}
\hline Normal & Less than 120/80 \\
Elevated & $120-129 / 80$ \\
High (stage 1) & $130-139 / 80-89$ \\
High (stage 2) & $\geq 140 / \geq 90$ \\
Hypertensive crisis & $>180 />120$ \\
\hline
\end{tabular}

\section{Statistical analysis}

The Kolmogorov-Smirnov test was used to assess the normality of data distribution. The preoperative and postoperative amylase and $\mathrm{SpO}_{2}$ values were compared using the Wilcoxon signed-rank, owing to their nonparametric distribution. The preoperative and postoperative $\mathrm{BP}$ and $\mathrm{HR}$ values were compared using the paired t-test, owing to their parametric distribution. The differences between the preoperative and postoperative values of all variables were calculated, and Spearman's correlation was performed to assess the correlation between the different variables. Statistical analysis was conducted using the IBM SPSS Statistical Software version 23 (Armonk, USA).

\section{Results}

Table 2 summarizes the descriptive statistics for the entire study sample.

A significant increase was observed in sAA after implant surgery $(p<0.001)$. On the other hand, the changes in the postoperative $\mathrm{HR}$ and $\mathrm{SpO}_{2}$ values were not significant ( $p=0.073$ and 0.102 , respectively). Only the systolic BP decreased significantly after surgery $(p=0.001)$ (Figs. 1, 2 and 3, Table 3).
Table 3 Paired comparison between the different parameters before and after surgery

\begin{tabular}{lllll}
\hline & Before & After & Difference & $\boldsymbol{p}$ value \\
\hline SAA & $10.8 \pm 22.5$ & $18.5 \pm 23$ & $-7.7 \pm 33.5$ & $<0.001^{*}$ \\
$\mathrm{HR}$ & $79.2 \pm 11.9$ & $75.2 \pm 11.9$ & $5.4 \pm 17.9$ & 0.073 \\
$\mathrm{SpO}_{2}$ & $96.9 \pm 1.6$ & $97.4 \pm 1.3$ & $4.7 \pm 12.3$ & 0.102 \\
$\mathrm{BP}$ (systolic) & $141.5 \pm 19.6$ & $136.1 \pm 18.3$ & $4 \pm 6.6$ & $0.001^{*}$ \\
$\mathrm{BP}$ (diastolic) & $82.1 \pm 13.5$ & $77.4 \pm 11.8$ & $-0.4 \pm 1.5$ & 0.084
\end{tabular}

*Significant

BP: blood pressure, sAA: salivary alpha-amylase, HR: heart rate, SpO2: peripheral blood oxygen saturation

A significant positive correlation was observed between sAA and HR $\left(r_{\mathrm{s}}=0.434, p=0.007\right)$ (Fig. $\left.4 \mathrm{~b}\right)$. A significant positive correlation was also observed between sAA and $\mathrm{SpO}_{2}\left(r_{s}=0.392, p=0.016\right)$ (Fig. 4a). Conversely, the correlation between sAA and BP was insignificant (systolic; $r_{s}=0.078, p=0.646$, diastolic; $r_{s}=0.195, p=0.247$ ) (Fig. 4c, d, Table 4).

Eight patients in this study exhibited high sAA activity on the follow-up measurement (i.e., after surgery), compared to only three patients who exhibited high sAA activity before surgery. This may be indicative of the increase in the patients' stress levels during surgery, which would be related to the surge in sAA activity.

\section{Discussion}

The activity of sAA is affected by several factors, which mainly include physical and psychological stressors. A study has reported that sAA activity increases in response to the acute stress induced by venepuncture during a periodic medical examination

Table 2 Descriptive statistics for the outcomes investigated in this study

\begin{tabular}{|c|c|c|c|c|c|}
\hline & & Before & & After & \\
\hline \multirow[t]{2}{*}{$\operatorname{Sex}[n(\%)]$} & Female & $24(64.9)$ & & $24(64.9)$ & \\
\hline & Male & $13(35.1)$ & & $13(35.1)$ & \\
\hline \multirow[t]{2}{*}{ Operation type $[n(\%)]$} & $1 s t$ & $17(45.9)$ & & $17(45.9)$ & \\
\hline & 2nd & $20(54.1)$ & & $20(54.1)$ & \\
\hline \multirow[t]{5}{*}{ Blood pressure [n(\%)] } & Normal & $4(10.8)$ & & $9(24.3)$ & \\
\hline & Elevated & $6(16.2)$ & High 33(89.2) & $6(16.2)$ & High 28 (78.7) \\
\hline & High (stage 1) & $7(18.9)$ & & $1(2.7)$ & \\
\hline & High (stage 2) & $17(45.9)$ & & $21(56.8)$ & \\
\hline & Hypertensive Crisis & $3(8.1)$ & & $0(0)$ & \\
\hline \multirow[t]{4}{*}{ Salivary alpha-amylase score [n(\%)] } & Score 1 & $34(91.9)$ & & $29(78.4)$ & \\
\hline & Score 2 & $2(5.4)$ & High 3 (8.1) & $1(2.7)$ & High 8 (21.6) \\
\hline & Score 3 & $0(0)$ & & $6(16.2)$ & \\
\hline & Score 4 & $1(2.7)$ & & $1(2.7)$ & \\
\hline Heart rate $[$ mean \pm SD] & & $79.2 \pm 11.9$ & & $75.2 \pm 11.9$ & \\
\hline Oxygen level [mean \pm SD] & & $96.9 \pm 1.6$ & & $97.4 \pm 1.3$ & \\
\hline
\end{tabular}




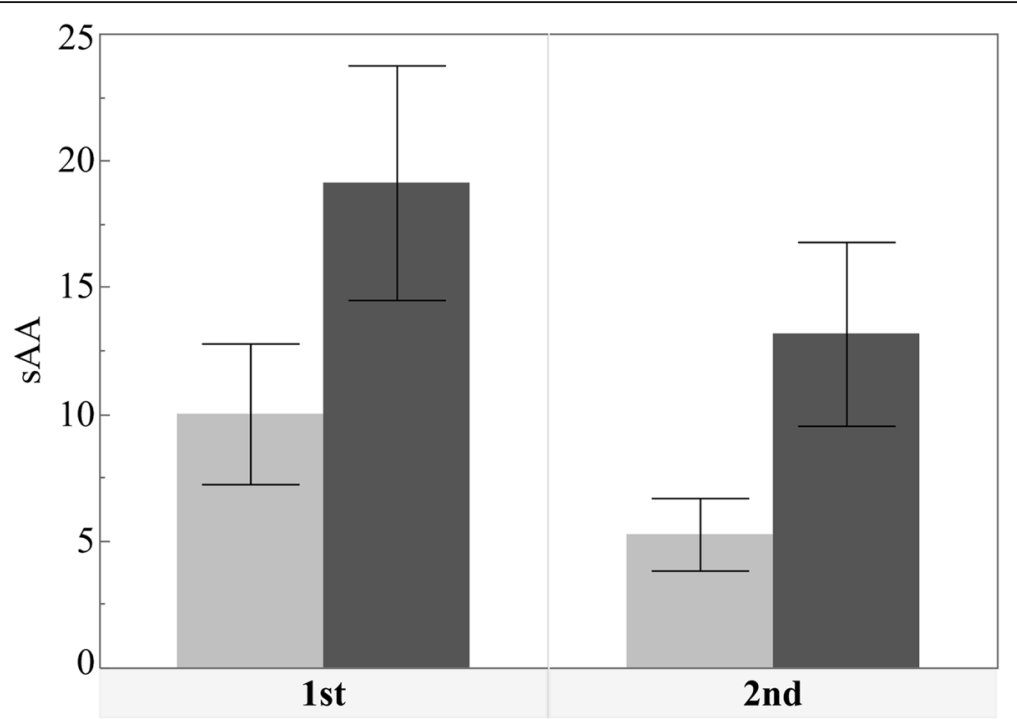

Fig. 1 Bar chart showing the mean SAA activity level before and after operation. A significant increase was seen in sAA after the operation $(p<0.001)$

and remains elevated $15 \mathrm{~min}$ after the procedure [21]. Another study reported significant differences in sAA, salivary cortisol, plasma catecholamines, and cardiovascular parameters between the stress and resting conditions [17]. Ali et al. reported that sAA activity was elevated during and even $15 \mathrm{~min}$ after venepuncture [21], even though venepuncture during periodic occupational health examinations is less invasive than the procedures used in dental implant-related surgeries. Similarly, our study found that sAA activity was elevated after surgery, even though the other vital signs returned to normal immediately. Thus, the results suggest that sAA activity is elevated because of the fear of pain or stress, and the elevation can last long enough to enable subsequent monitoring. This implies that sAA activity can be used as an additional method to monitor the patient's history of mental and physical stress or the accumulation of such stress perioperatively.

Invasive major surgeries are usually performed under general anesthesia, which means that the patient is unconscious and does not experience fear of pain or

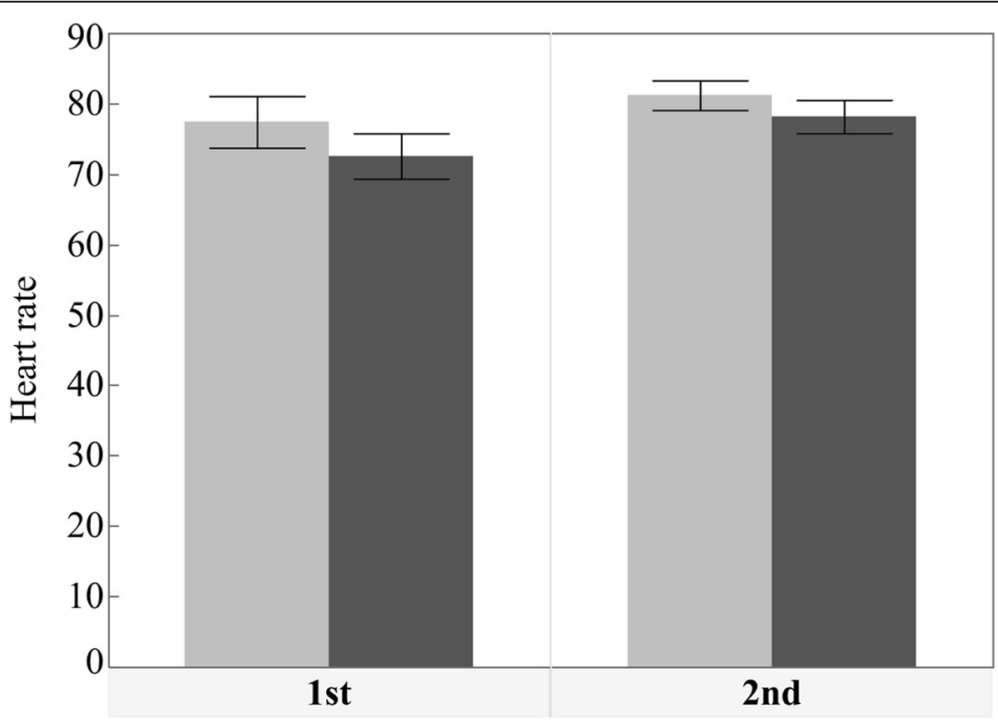

Fig. 2 Bar chart showing the mean HR scores before and after operation 


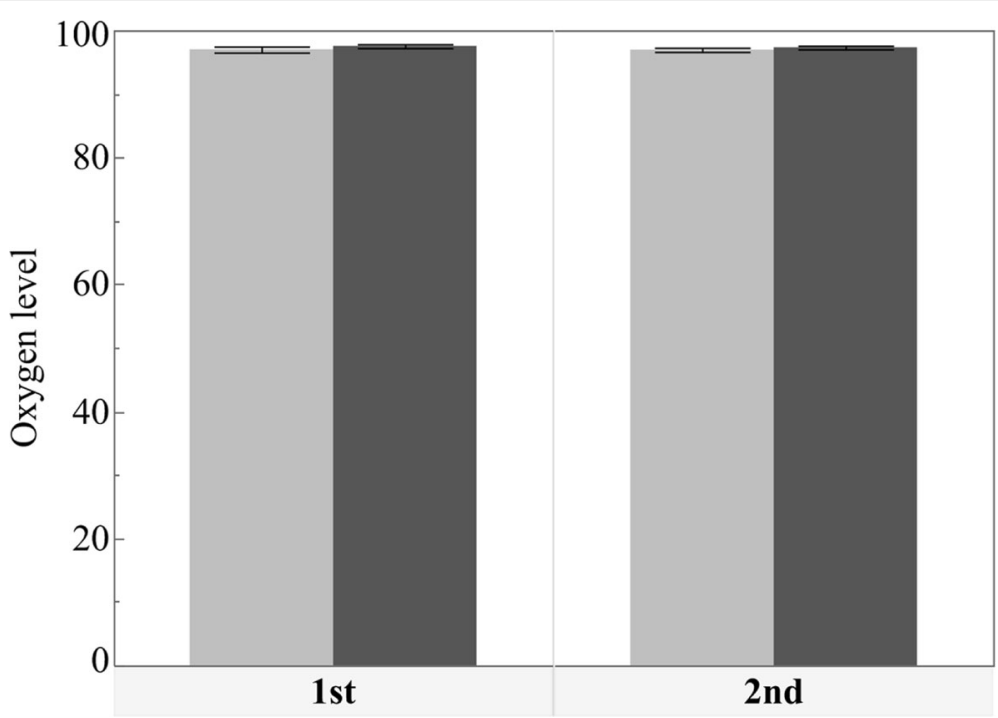

Fig. $3 \mathrm{Bar}$ chart showing the mean $\mathrm{SpO}_{2}$ scores before and after operation

mental stress during the procedure, irrespective of the operative time, whereas the patient must keep his/her mouth open during dental surgery, and the sound and sensation of drilling in the jawbone may cause a certain level of stress or fear since the patient is completely or partially conscious. Additionally, the patients cannot see their oral cavity during these procedures, which may induce severe anxiety in some individuals. We opine that evaluating the patient's anxiety fosters improvement in the preoperative informed consent taking activity and the surgical environment.

In this study, sAA activity was elevated predominantly in the postoperative measurement, which may be attributed to several factors. The duration of surgery may be one such factor, which may affect the stress level of the patient. Another factor may be related to patient exhaustion: if the surgery is long, the patient may get tired, causing the stress level to rise [22]. Furthermore, the proportion of patients with high sAA activity ( $>31$ : somewhat stressed level in

Table 4 Correlation between the differences in the observed parameters after surgery

\begin{tabular}{cccll}
\hline & $\mathrm{HR}$ & $\mathrm{SpO}_{2}$ & $\begin{array}{l}\mathrm{BP} \\
\text { (systolic) }\end{array}$ & $\begin{array}{l}\mathrm{BP} \\
\text { (diastolic) }\end{array}$ \\
\hline \begin{tabular}{l} 
SAA $\begin{array}{l}\text { Correlation } \\
\text { coefficient } \\
p \text { value }\end{array}$ \\
\multicolumn{1}{c}{$p$}
\end{tabular} & 0.434 & 0.392 & 0.078 & 0.195 \\
\hline
\end{tabular}

"Significant

HR: hear rate, SAA: salivary alpha-amylase, BP: blood pressure, SpO2: peripheral blood oxygen saturation accordance with Table 5) was greater after surgery than that before surgery. This may be representative of the surge in the patient's stress level during surgery, which would be related to an increase in sAA activity. We also observed a significant correlation between sAA activity and HR, indicating that it may be a valuable biomarker for evaluating stress and anxiety in dental patients [23].

One of the patients developed a hypertensive crisis, which led to cancellation of surgery and exclusion of data from the final analysis. We measured his sAA activity, which was $69 \mathrm{kIU} / \mathrm{L}$ (very stressed, based on Table 1). We assumed that this patient was very stressed, and his $\mathrm{BP}$ and sAA activity may have been correlated and increased due to anxiety and stress [5]. However, our study did not find any statistically significant correlation between sAA and BP.

We could not determine whether intravenous or local anesthesia had any effect on sAA, owing to the limited sample size of our study. However, most patients who underwent intravenous anesthesia exhibited an increase in the sAA activity during the postoperative measurement. Furthermore, our results showed an increase in the overall postoperative measurement of SAA activity. No difference was detected with respect to the type and duration of surgery.

In our study, the operative time was higher for the first surgery (median $=56.5$ and range $=35 \mathrm{~min}$ ) compared to the second surgery (median $=27$ and range $=15.8 \mathrm{~min}$ ). The operative time was excluded from comparison, as was the comparison between the first and second surgeries. Our principal aim was to monitor sAA activity and 

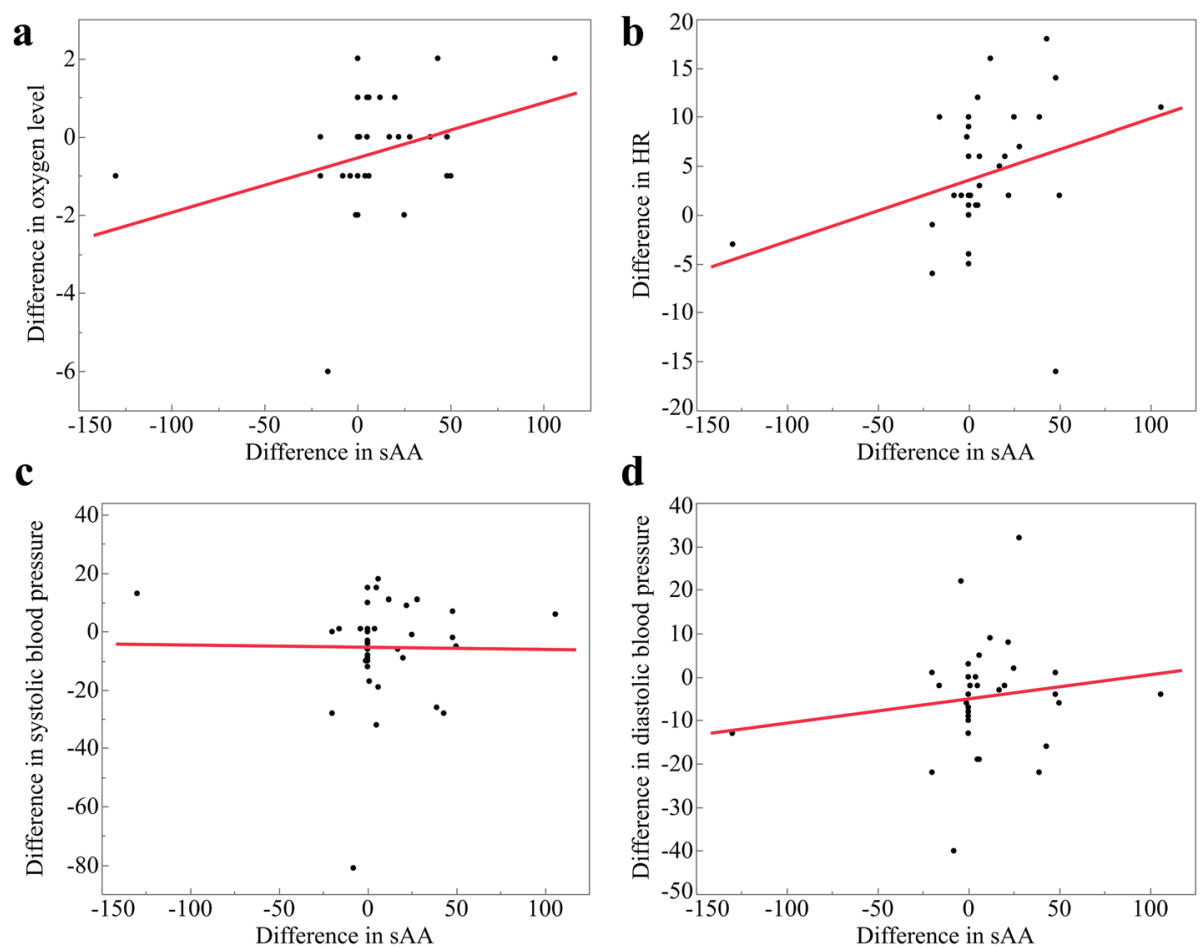

Fig. 4 Scatter plot showing the correlation between the difference of sAA and a oxygen level, b heart rate, $\mathbf{c}$ systolic blood pressure, and $\mathbf{d}$ diastolic blood pressure. A positive correlation presented between difference in SAA and $\mathbf{a}$ oxygen level and $\mathbf{b}$ heart rate $(p<0.05)$

its correlation with other measurable variables as a general effect, irrespective of other variables.

Further studies are required to evaluate the difference in sAA activity caused by the operative time, surgical procedure, sex-based differences, and types of anesthesia.

\section{Conclusion}

This study found a significant positive correlation between sAA activity and HR; furthermore, $\mathrm{SpO}_{2}$ and sAA activity also showed a significant positive correlation.

Our results found no correlation between BP and sAA. Finally, sAA activity may provide additional information to monitor the history of the patient's mental and physical stress or the accumulation of such stress during dental implant surgery.

Table 5 Reference values for salivary alpha-amylase

\begin{tabular}{ll}
\hline Results (kIU/L) & Stress level \\
\hline $0-30$ & None \\
$31-45$ & Somewhat stressed \\
$46-60$ & Stressed \\
$>61$ & Very stressed \\
\hline
\end{tabular}

\section{Abbreviations}

ANS: Autonomic nervous system; LA: Local anesthesia; IV: Intravenous anesthesia; PSNANS: Parasympathetic nervous system; sAA: Salivary al phaamylase; SNS: Sympathetic nervous system; $\mathrm{SpO}_{2}$ : Peripheral blood oxygen saturation

\section{Acknowledgements}

The authors wish to thank the Department of Oral Implantology \& Regenerative Dental Medicine and colleagues for their cooperation.

\section{Authors' contributions}

A.S., H.N., and S.K. contributed to the conception of the study. A.S. and H.N acquired the data. A.S., H.N., and A.A analyzed the data. A.S. and H.N. drafted the manuscript and revised it. S.K. and S.K. revised the final version. All authors have read and approved the final manuscript.

\section{Author's information}

Not applicable.

\section{Funding}

This study was supported by the Tokyo Medical and Dental University, PhD program.

Availability of data and materials

The datasets used and/or analyzed during the current study are available from the corresponding author on reasonable request.

\section{Declarations}

\section{Ethics approval and consent to participate}

This study was approved by the ethical committee of Tokyo Medical and Dental University under approval numbers D2020-027 and D2020-028. The purpose of the study was explained to all patients, and informed consent was obtained. 


\section{Consent for publication}

Not applicable.

\section{Competing interests}

Afnan Sabbagh, Hidemi Nakata, Ahmed Abdou, Shohei Kasugai and Shinji Kuroda declare that they have no competing interests.

\section{Author details}

'Department of Oral Implantology and Regenerative Dental Medicine, Division of Oral Health Sciences, Graduate School of Medical and Dental Sciences, Tokyo Medical and Dental University, 1-5-45 Yushima, Bunkyo-ku, Tokyo 113-8510, Japan. ${ }^{2}$ Department of Cariology \& Operative Dentistry, Graduate School of Medical and Dental Sciences, Tokyo Medical and Dental University, 1-5-45 Yushima, Bunkyo-ku, Tokyo 113-8549, Japan.

Received: 6 October 2020 Accepted: 31 March 2021

Published online: 29 June 2021

\section{References}

1. Lopes Brilhante Bhering C, Feitosa Henriques SE, da Silva Girundi FM, Edson Miranda M, Araújo de Vasconcellos A. Full arch implant supported oral rehabilitation to reestablish esthetic and function. Int J Clin Dent. 2017;10: 25-33.

2. Shigehara S, Ohba S, Nakashima K, Takanashi Y, Asahina I. Immediate loading of dental implants inserted in edentulous maxillas and mandibles: 5-year results of a clinical study. J Oral Implantol. 2015;41(6):701-5 https:// doi.org/10.1563/aaid-joi-D-14-00018.

3. Kohli S, Bhatia S, Kaur A, Rathakrishnan T. Patients awareness and attitude towards dental implants. Indian J Dent. 2015;6(4):167-71 https://doi.org/1 0.4103/0975-962X.168518.

4. Cha H-S, Kim J-W, Hwang J-H, Ahn K-M. Frequency of bone graft in implant surgery. Maxillofac Plast Reconstr Surg. 2016;38(1) https://doi.org/10.1186/s4 0902-016-0064-2.

5. Wada M, Miwa S, Mameno T, Suganami T, Ikebe K, Maeda Y. A prospective study of the relationship between patient character and blood pressure in dental implant surgery. Int J Implant Dent. 2016;38(1):19 https://doi.org/1 0.1186/s40902-016-0064-2.

6. Coulthard P. Should GDPs be checking blood pressure? Br Dent J. 2002; 193(5):269 https://doi.org/10.1038/sj.bdj.4801542.

7. Southerland JH, Gill DG, Gangula PR, Halpern LR, Cardona CY, Mouton CP. Dental management in patients with hypertension: challenges and solutions. Clin Cosmet Investig Dent. 2016;8:111-20 https://doi.org/10.2147/ CCIDE.S99446.

8. Yagiela JA, Haymore TL. Management of the hypertensive dental patient. J Calif Dent Assoc. 2007:35(1):51-9.

9. Skaret E, Raadal M, Berg E, Kvale G. Dental anxiety and dental avoidance among 12 to 18 year olds in Norway. Eur J Oral Sci. 1999;107(6):422-8 https://doi.org/10.1046/j.0909-8836.1999.eos107602.x.

10. Waxenbaum JA, Varacallo M. Anatomy, Autonomic Nervous System. In: StatPearls. Treasure Island: StatPearls Publishing; 2019. https://www.ncbi.nlm. nih.gov/books/NBK539845/.

11. Bergström RM. Physiology of the autonomic nervous system. Acta Anaesthesiol Scand. 1964;8:17-20 https://doi.org/10.1111/j.1399-6576.1964. tb00252.x.

12. Spielberger CD, Sarason IG, Strelau J, Brebner JM, editors. Stress And Anxiety. New York: Taylor \& Francis; 1991. https://doi.org/10.4324/978131 5800851

13. Bouayed J, Rammal H, Soulimani R. Oxidative stress and anxiety: relationship and cellular pathways. Oxid Med Cell Longev. 2009;2(2):63-7 https://doi. org/10.4161/oxim.2.2.7944.

14. Möstl E, Palme R. Hormones as indicators of stress. Domest Anim Endocrinol. 2002;23(1-2):67-74 https://doi.org/10.1016/S0739-7240(02)00146-7.

15. Hellhammer DH, Wüst $\mathrm{S}$, Kudielka BM. Salivary cortisol as a biomarker in stress research. Psychoneuroendocrinology. 2009;34(2):163-71 https://doi. org/10.1016/j.psyneuen.2008.10.026

16. Hong HR, Oh Yl, Kim YJ, Seo KW. Salivary alpha-amylase as a stress biomarker in diseased dogs. J Vet Sci. 2019;20(5):e46 https://doi.org/10.4142/ jvs.2019.20.e46.

17. Nater UM, La Marca R, Florin L, Moses A, Langhans W, Koller MM, et al. Stress-induced changes in human salivary alpha-amylase activity - associations with adrenergic activity. Psychoneuroendocrinology. 2006;31(1): 49-58 https://doi.org/10.1016/j.psyneuen.2005.05.010.

18. Van Stegeren A, Rohleder N, Everaerd W, Wolf OT. Salivary alpha amylase as marker for adrenergic activity during stress: effect of betablockade. Psychoneuroendocrinology. 2006;31(1):137-41 https://doi.org/10.1016/j. psyneuen.2005.05.012

19. Noto Y, Sato T, Kudo M, Kurata K, Hirota K. The relationship between salivary biomarkers and state-trait anxiety inventory score under mental arithmetic stress: a pilot study. Anesth Analg. 2005:1873-6 https://doi.org/10.1213/01.A NE.0000184196.60838.8D.

20. Shimazaki M, Matsuki T, Yamauchi K, Iwata M, Takahashi H, Sakamoto K, et al. Clinical performance of a salivary amylase activity monitor during hemodialysis treatment. Biomark Insights. 2008;3:BMI.S997 https://doi.org/1 0.4137/bmi.s997.

21. Koh $\mathrm{D}, \mathrm{Ng} \mathrm{V}$, Naing L. Alpha amylase as a salivary biomarker of acute stress of venepuncture from periodic medical examinations. Front Public Heal. 2014;2 https://doi.org/10.3389/fpubh.2014.00121.

22. Ali N, Nater UM. Salivary alpha-amylase as a biomarker of stress in behavioral medicine. Int J Behav Med. 2020;27(3):337-42 https://doi.org/10.1 007/s12529-019-09843-X.

23. Furlan NF, Gavião MBD, Barbosa TS, Nicolau J, Castelo PM. Salivary cortisol, alpha-amylase and heart rate variation in response to dental treatment in children. J Clin Pediatr Dent. 2012; https://doi.org/10.17796/jcpd.37.1.n32 m21n08417v363.

\section{Publisher's Note}

Springer Nature remains neutral with regard to jurisdictional claims in published maps and institutional affiliations.

\section{Submit your manuscript to a SpringerOpen ${ }^{\circ}$ journal and benefit from:}

- Convenient online submission

- Rigorous peer review

- Open access: articles freely available online

- High visibility within the field

- Retaining the copyright to your article

Submit your next manuscript at $>$ springeropen.com 